\title{
Learning Curve for Percutaneous Endoscopic Lumbar Discectomy
}

\author{
Dong Yeob LEE and Sang-Ho LEE
}

Department of Neurosurgery, Wooridul Spine Hospital, Seoul, R.O.K.

\begin{abstract}
Percutaneous endoscopic lumbar discectomy (PELD) is one of the surgical options for soft lumbar disk herniation, but the learning curve is perceived to be steep. The first 51 PELD cases performed for singlelevel intracanalicular lumbar disk herniation causing radiculopathy by the same surgeon were prospectively studied. The patients were divided into 3 groups of 17 patients, and the PELD learning curve was assessed by evaluating operating time, failure rate, complication rate, and 1-year reherniation rate. One-year clinical success rate was assessed by telephone interviews. The herniated disk was successfully removed by PELD in 47 patients. Four patients required subsequent open discectomy due to PELD failure. There were 2 minor complications. One year after surgery, clinical success was achieved in 42 of the 47 patients in whom PELD was initially successful, and reherniation developed in 5 patients. A significant reduction in operating time was observed after 17 patients had been treated $(p=0.0004)$. No significant differences were observed in terms of either failure rate or complication rate between the 3 groups. No significant differences were observed in terms of either the clinical success rate or the reherniation rate at 1 year after surgery. The PELD learning curve seems to be stable and acceptable with proper pre-PELD training.
\end{abstract}

Key words: disk herniation, learning curve, percutaneous endoscopic lumbar discectomy

\section{Introduction}

Percutaneous endoscopic lumbar discectomy (PELD) has recently been performed as an alternative to classic open discectomy with results that are comparable to those of open discectomy. ${ }^{1,2,4,6,8,9,11,13,14,18,20,22,25,26)}$ PELD has several advantages over open discectomy ${ }^{1,9,15,23)}$; PELD is usually performed under local anesthesia, postoperative pain is quite minimal, normal paraspinal structures are preserved, and the risk of postoperative epidural scar formation and instability can be minimized. However, the PELD learning curve is usually perceived to be steep. ${ }^{7,15,26)}$ Furthermore, despite the low complication rate, major complications such as neurovascular injury, visceral injury, and/or spondylodiscitis may occur, possibly resulting from lack of skilled surgical techniques during the learning period. ${ }^{12,19,21,26)}$

The present study profiles the PELD learning curve of an individual surgeon not previously exposed to this procedure.

Received November 19, 2007; Accepted June 5, 2008

\section{Materials and Methods}

\section{Patient population}

The first 51 consecutive PELD procedures performed by the same surgeon (D.Y.L.) for lumbar disk herniation causing radiculopathy between November 2004 and October 2005 were prospectively studied. He had performed about 200 cases of open microdiscectomy, but had no previous experience with percutaneous endoscopic procedures. Throughout this prospective study, the indication for PELD was a single level intracanalicular soft lumbar disk herniation of the lower lumbar vertebrae, which was unresponsive to conservative therapy. Conventional open discectomy was performed in patients with hard disk herniation, foraminal and extraforaminal disk herniations, sequestrated fragment, and/or severe neurological deficits. Spinal fusion was first considered in patients with spinal instability.

The 32 males and 19 females were aged 17-55 years (mean 36.4 years). Thirty-nine of the 51 patients presented with low back pain and leg pain, and 12 with leg pain only. Twelve patients also showed motor weakness on great toe dorsiflexion (power grade IV in 11 patients and III in 1 patient). 
The mean duration of symptoms was 4.8 months (range 0.3-24 months). The PELD spinal levels were L4-5 in 46 patients and L5-S1 in 5 patients. All 5 patients who underwent PELD at the L5-S1 level had a low-lying iliac crest on radiography. Three patients underwent PELD for recurrent disk herniation.

The patients were divided into 3 groups of 17 patients (Group I: Cases 1-17, Group II: Cases 18-34, and Group III: Cases 35-51), and the operating time, failure rate, complication rate, 1-year clinical success rate, and 1-year reherniation rate were evaluated. Reherniation was defined as a disk herniation occurring ipsilaterally at the same level as a previously operated lumbar disk, as demonstrated on follow-up magnetic resonance (MR) imaging, with a pain-free interval after the primary discectomy of greater than 4 weeks. Clinical success rate was analyzed 1 year after surgery by telephone interview. Clinical success was defined as $\geq 2$ point improvement in Visual Analogue Scale (VAS) score, $\geq 25 \%$ improvement in Oswestry Disability Index (ODI) score, $\geq 50 \%$ patient's subjective recovery rate, and no major complication related to the surgery. ${ }^{24)}$ The analysis of variance (ANOVA) test, chi-square test, Kruskal-Wallis test, Wilcoxon rank sum test, and Fisher's exact test were used to compare the differences of each parameter between the 3 groups using SAS software (version 8.1; SAS Institute, Cary, N.C., U.S.A.). $P$ values less than 0.05 were considered significant.

\section{Surgical technique}

Before starting this prospective study, the surgeon observed 15 PELD procedures performed by experienced surgeons and performed about 40 epidural block procedures via the transforaminal route during a 3-month training period. The surgeon also attended two PELD workshops; one with a silicon model and the other with a cadaver.

All operations followed a standard pattern suggested by the senior author. ${ }^{2,15}$ The procedure was performed under local anesthesia in the prone position on a radiolucent table. Before the beginning of the procedure, patients were informed about all steps of the procedure. Patients could communicate with the surgeon during the entire procedure. The skin entry point was usually about $10-12 \mathrm{~cm}$ from the midline. After infiltration of the entry point with local anesthetics, an 18-gauge spinal needle was introduced under fluoroscopy guidance. The final target point of the spinal needle was the medial pedicular line on the anteroposterior image and the posterior vertebral line on the lateral image. Then epidurography was performed using contrast medi-

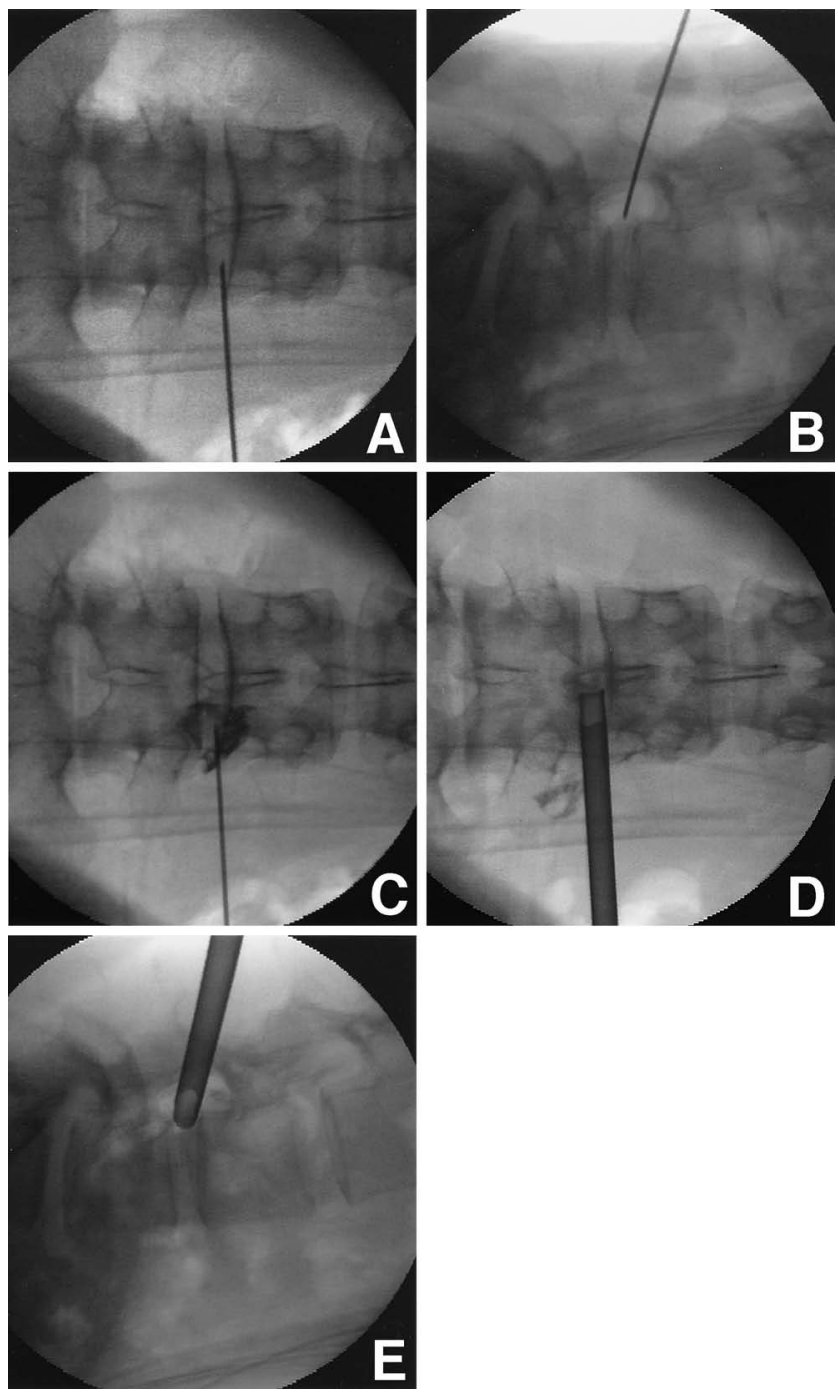

Fig. 1 Anteroposterior fluoroscopic image (A) showing the final target point of the initial approach step on the medial pedicular line, and lateral fluoroscopic image (B) showing the posterior vertebral line. Epidurogram (C) demonstrating the safe triangular working zone. Anteroposterior (D) and lateral (E) fluoroscopic images taken after insertion of the endoscopic cannula.

um to confirm the location of the exiting root and the traversing root. After insertion of the spinal needle into the disk, the nucleus pulposus was stained blue with $1 \mathrm{ml}$ of indigo carmine. Then a guide wire was inserted through the spinal needle, the spinal needle was removed, a small skin incision was made at the entry site, a tapered cannulated obturator was inserted along the guide wire, the obturator was inserted into the disk with hammering after touching the annulus, and a bevel-ended, oval-shaped work- 

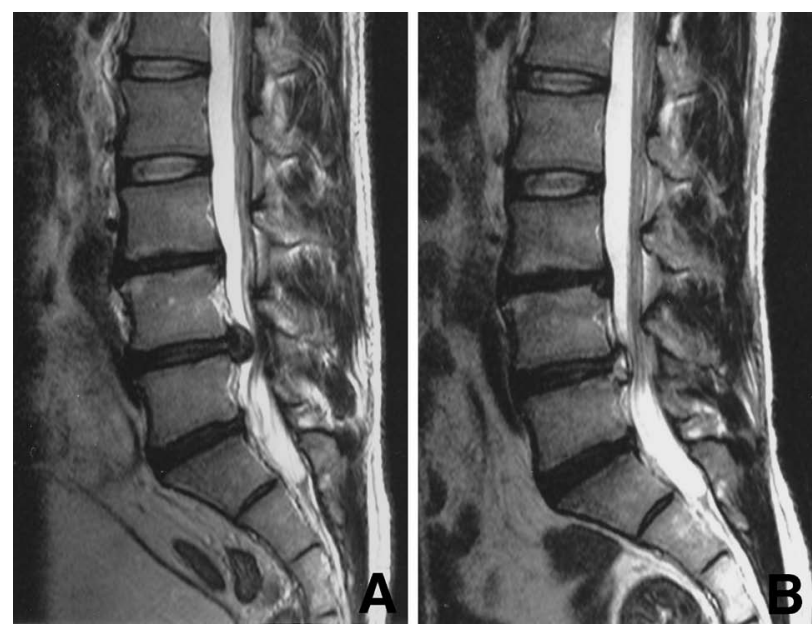

Fig. 2 Preoperative (A) and postoperative (B) $\mathrm{T}_{2^{-}}$ weighted magnetic resonance images demonstrating successful removal of the herniated disk at the L4-5 level by percutaneous endoscopic lumbar discectomy.

ing cannula was inserted into the disk along the obturator, and then the obturator was removed (Fig. 1).

The endoscope was then inserted through the cannula. The blue-stained disk was removed using small forceps and a side firing holmium yttriumaluminum-garnet (Ho:YAG) laser using the in and out' technique, working from the central portion to the lateral portion of the disk space on the anteroposterior image. Targeted fragmentectomy was performed in all cases. The herniated fragment was removed, the endoscope was removed, and a sterile dressing was applied with a 1-point suture. All patients underwent MR imaging immediately after the procedure to confirm adequate decompression (Fig. 2). If the patient's symptoms had improved and adequate decompression was confirmed by MR imaging, the patient was usually discharged within 12 hours after the procedure.

\section{Results}

\section{Clinical outcomes}

The operating time was 30-105 minutes (mean 49.2 minutes). The herniated disks were successfully removed by PELD in 47 patients (92.2\%), and all these patients showed marked improvement in their symptoms immediately after surgery. PELD failed in 4 patients $(7.8 \%$; Cases 27, 29, 33, and 41$)$, caused by failure to remove small disk fragments located in the lateral recess in 2 patients, failure to remove a centrally protruding huge disk with a narrow base in 1 patient, and an unrecognized newly developed migrated disk, which apparently occurred just before the operation, in 1 patient. All these 4 patients subsequently underwent open discectomy. Two minor complications $(3.9 \%)$ occurred in the entire series. Case 11 complained of dysesthesia on the anterolateral thigh after the PELD procedure at the L4-5 level, which improved spontaneously 2 days after surgery. Case 22 showed pain shock during the procedure (just after annulotomy with the obturator) and was successfully managed with medical treatment. There were no significant major complications, such as neurovascular injury or significant infection.

One-year surgical outcomes were assessed in the 47 patients with initially successful PELD. The mean preoperative VAS score was 5.5 (range 5-10) and improved significantly at 1 year after surgery to 1.2 (range $0-5)(\mathrm{p}<0.001)$. The mean preoperative ODI score was $57.9 \%$ (range $28.9-90 \%$ ) and improved significantly at 1 year after surgery to $15.9 \%$ (range $0-46.7 \%)(p<0.001)$. The mean subjective recovery rate at 1 year after surgery was $80.7 \%$ (range $20-100 \%$ ). Clinical success was achieved in 42 of 47 patients (89.4\%), but not in Cases 5, 10, 11, 19, and 47 at 1 year after surgery. Reherniation developed in 5 patients (10.6\%; Cases 5, 10, 17, 31, and 38) within 1 year after surgery. The mean interval to recurrence was 6.4 months (range 4-10 months). Two patients underwent additional surgery, open discectomy in Case 5 and repeated PELD in Case 10, and the other 3 improved after conservative treatment.

\section{Learning curve}

The mean age of the patients was similar in the 3 groups (ANOVA, $p=0.20$ ). The sex ratio was also similar in the 3 groups (chi-square test, $\mathrm{p}=0.72$ ). The mean operating time was 62.1 minutes for Group I, 47.6 minutes for Group II, and 37.9 minutes for Group III, showing a significant difference (Kruskal-Wallis test, $\mathrm{p}=0.0004$ ). The mean operating time for Group I, which contained the earliest 17 cases, was significantly longer than those for Groups II and III (Wilcoxon rank sum test, Group I vs. II, p $=0.02$; Group I vs. III, p $=0.0005$; Group II vs. III, $\mathrm{p}=0.22$ ). PELD failed in 3 patients in Group II and 1 patient in Group III. There was no significant difference in the failure rates between the 3 groups (Fisher's exact test, $p=0.31$ ). Minor complications occurred in 1 patient in Group I and 1 patient in Group II. There was no significant difference in the complication rates between the 3 groups (Fisher's exact test, $\mathrm{p}=1.0$ ). The 1 -year clinical success rate was $82.4 \%$ for Group I, $92.9 \%$ for Group II, and 93.8\% for Group III, with no significant difference (Fisher's exact test, $\mathrm{p}=0.51$ ). The 1-year rehernia- 
Table 1 Summary of factors affecting the learning curve

\begin{tabular}{|c|c|c|c|c|c|c|c|c|}
\hline & $\begin{array}{l}\text { Case } \\
\text { No. }\end{array}$ & $\begin{array}{l}\text { Mean age } \\
\text { (range), } \\
\text { yrs }\end{array}$ & $\begin{array}{c}\text { Male/ } \\
\text { female }\end{array}$ & $\begin{array}{l}\text { Operating time } \\
\text { (range) } \\
\text { min }\end{array}$ & $\begin{array}{l}\text { Failure } \\
\text { rate, \% }\end{array}$ & $\begin{array}{l}\text { Complication } \\
\text { rate, \% }\end{array}$ & $\begin{array}{l}\text { 1-Year } \\
\text { clinical } \\
\text { success } \\
\text { rate, \%* }\end{array}$ & $\begin{array}{l}\text { 1-Year } \\
\text { reherniation } \\
\text { rate, \%* }\end{array}$ \\
\hline Group I & $1-17$ & $33.9(24-52)$ & $10 / 7$ & $62.1(30-90)$ & 0 & 5.9 & 82.4 & 17.6 \\
\hline Group II & $18-34$ & $35.6(18-49)$ & $10 / 7$ & $47.6(30-105)$ & 17.6 & 5.9 & 92.9 & 7.1 \\
\hline Group III & $35-51$ & $39.8(15-55)$ & $12 / 5$ & $37.9(30-60)$ & 5.9 & 0 & 93.8 & 6.3 \\
\hline p Value & & 0.20 & 0.72 & 0.0004 & 0.31 & 1.0 & 0.51 & 0.60 \\
\hline
\end{tabular}

${ }^{*}$ The 1-year clinical success rate and 1-year reherniation rate were assessed in 47 patients with initial success of percutaneous endoscopic lumbar discectomy.

tion rate was $17.6 \%$ for Group I, $7.1 \%$ for Group II, and $6.3 \%$ for Group III, with no significant difference (Fisher's exact test, $p=0.60$ ). A summary of each factor concerning the learning curve is shown in Table 1.

\section{Discussion}

The surgical techniques for percutaneous endoscopic discectomy have rapidly evolved since the introduction of posterolateral percutaneous disk decompression. ${ }^{10)}$ The main concept of the procedure has changed from indirect decompression of the central disk to direct targeted fragmentectomy. ${ }^{2,15)} \mathrm{A}$ prospective study on the surgical outcome of the first consecutive 42 patients who underwent PELD via the transforaminal route found a high failure rate of 14/42, of whom 7 patients eventually underwent re-operation (open discectomy), and 6 of these 7 patients in the first 8 months. ${ }^{7)}$ Therefore, the PELD technique has a steep learning curve, which can be overcome with training and suitable patient selection.

The present study indicated that the PELD learning curve is acceptable, with relatively low failure and complication rates of $7.8 \%$ and $3.9 \%$, respectively, and no significant major complications. Comparison of the early, middle, and late experience groups found no significant differences in the factors concerning the learning curve except operating time. Targeted fragmentectomy was performed in all patients.

One of the most important surgical steps of PELD is the initial approach, which places the spinal needle exactly at the final target point along the optimal trajectory. The tip of the spinal needle touching the annulus should be located at the medial pedicular line on the anteroposterior fluoroscopic image and the posterior vertebral line on the lateral fluoroscopic image. Failure to place the spinal needle at the optimal target point usually results in the placement of the endoscopic cannula at an inappropriate location, which almost always results in failure to remove the herniated disk. Another related problem is the possibility of nerve root injury during annulotomy and/or cannular insertion. If the tip of the spinal needle is exactly located at the optimal target point, the spinal needle is always located between the exiting root and traversing root, which can be confirmed by epidurography. Then, annulotomy followed by cannular insertion can be performed safely. Therefore, the surgeon should develop high needle technique before starting PELD, and so prePELD training was important in the stable learning curve of the present study. We recommend performing an epidural block via the transforaminal route before starting PELD, which is a very similar procedure to the initial PELD procedure step and enables beginners to develop the needle technique for PELD. Participating in PELD workshops is another way to develop needle technique and other core skills essential for PELD.

In general, correct patient selection is considered paramount to ensure satisfactory outcome after PELD. Several highly experienced endoscopic surgeons have recently expanded the indications for PELD and have reported successful results even in patients with migrated disk herniation and foraminal/extraforaminal disk herniation. ${ }^{6,16)}$ However, PELD surgical outcomes are affected by the surgeon's skill and personal technique in such situations. ${ }^{2,15)}$ Furthermore, especially for inexperienced surgeons, improper selection of patients often results in incomplete decompression and the necessity for subsequent open discectomy, and this can sometimes result in a poor surgical outcome. ${ }^{15)}$ Retrospective analysis of 1586 patients who had undergone PELD for intracanalicular disk herniation concluded that open surgery may be considered for herniations with high canal compromise and highgrade migrations. ${ }^{17)}$

In the present study, the authors adopted narrow 
inclusion criteria: single-level intracanalicular soft lumbar disk herniation at the L4-5 level and L5-S1 level (with low-lying iliac crest). Patients with hard disk herniation, foraminal and extraforaminal disk herniations, sequestrated fragments, severe neurological deficits, and spinal instability were excluded. The surgical outcomes of the present study were satisfactory, with an immediate success rate and 1year clinical success rate of $92.2 \%$ and $89.4 \%$, respectively. One notable finding in the present study is that PELD failure occurred after 26 cases were treated: 3 cases in Group II and 1 case in Group III. Although the narrow inclusion criteria were retained throughout the study, a progressive increase in the difficulty of the cases might have resulted in this phenomenon.

One of the concerns for the PELD learning curve is perioperative complication. Serious complications during the PELD learning period are rare but may lead to profound morbidity. In this regard, approaching the safe triangular zone via the optimal trajectory is of utmost importance. An inappropriate approach may increase the risk for neurovascular or visceral injury. Inadvertent contact of the spinal needle with the abdominal viscera might result in psoas abscess and/or spondylodiscitis. A case of postoperative psoas abscess with spondylodiscitis occurred after PELD. ${ }^{12)}$ The isolated organism was Escherichia coli, suggesting that inadvertent tap into the colon during the initial needle approach to the disk space was the cause. Meticulous preoperative planning with careful review of axial computed tomography and MR imaging is recommended. The complication rate of the present study, only 2 minor complications, is acceptable and comparable to those of experienced surgeons.,26) Temporary dysesthesia is the most commonly reported complication after PELD and is usually provoked by irritation of the exiting root or traversing root during the operation, which can be prevented by confirmation of the safe triangular working zone and careful monitoring of the patient during operation. ${ }^{15)}$ Pain shock, vasovagal syncope due to severe pain, can happen during PELD, especially during annulotomy and/or cannular insertion, as in our study. Nowadays, the authors prevent pain shock by additionally injecting 1 or $2 \mathrm{~cm}^{3}$ of lidocaine on the surface of the annulus just before the annulotomy.

In the present study, the 1-year reherniation rate was relatively high $(10.6 \%)$. Few authors have stated concerns about reherniation after PELD. ${ }^{5,15)}$ In a previous study, in which experienced senior surgeons performed all procedures, the reherniation rate after PELD was $2.2 \%$ at a mean follow up of 37.2 months. ${ }^{15)}$ Recently, a prospective randomized study on transforaminal posterolateral endoscopic discectomy with or without the combination of low-dose chymopapain found the endoscopy only group had a reherniation rate of $6.9 \%$, and the combination group had a recurrence rate of $1.6 \%$ at 1 -year postoperatively. ${ }^{5)}$ The relatively high reherniation rate of the present study might be attributable to targeted fragmentectomy, a concept similar to limited discectomy in conventional open discectomy. The reherniation rate in the limited discectomy group $(18 \%)$ was higher than in the subtotal discectomy group (9\%) at 2-year follow up. ${ }^{3)}$ Another influence on the 1-year reherniation rate is the learning curve. Group I, which contained the earliest 17 cases, showed a higher reherniation rate $(17.6 \%)$ than Groups II (7.1\%) and III (6.3\%). Though statistically not significant, these results suggest that increasing experience with PELD might influence the 1-year recurrence rate.

\section{Acknowledgment}

This study was supported by a grant from the Wooridul Spine Foundation.

\section{References}

1) Ahn Y, Lee SH, Park WM, Lee HY: Posterolateral percutaneous endoscopic lumbar foraminotomy for L5S1 foraminal or lateral exit zone stenosis. Technical note. J Neurosurg 99: 320-323, 2003

2) Ahn Y, Lee SH, Park WM, Lee HY, Shin SW, Kang HY: Percutaneous endoscopic lumbar discectomy for recurrent disc herniation: surgical technique, outcome, and prognostic factors of 43 consecutive cases. Spine 29: E326-E332, 2004

3) Carragee EJ, Spinnickie AO, Alamin TF, Paragioudakis S: A prospective controlled study of limited versus subtotal posterior discectomy: short-term outcomes in patients with herniated lumbar intervertebral discs and large posterior annular defect. Spine 31: 653-657, 2006

4) Hermantin FU, Peters T, Quartararo L, Kambin P: A prospective, randomized study comparing the results of open discectomy with those of video-assisted arthroscopic microdiscectomy. J Bone Joint Surg Am 81: 958-965, 1999

5) Hoogland T, Schubert M, Miklitz B, Ramirez A: Transforaminal posterolateral endoscopic discectomy with or without the combination of a low-dose chymopapain: a prospective randomized study in 280 consecutive cases. Spine 24: E890-E897, 2006

6) Jang JS, An SH, Lee SH: Transforaminal percutaneous endoscopic discectomy in the treatment of foraminal and extraforaminal lumbar disc herniations. J Spinal Disord Tech 19: 338-343, 2006

7) Kafadar A, Kahraman S, Akboru M: Percutaneous 
endoscopic transforaminal lumbar discectomy: a critical appraisal. Minim Invasive Neurosurg 49: 74-79, 2006

8) Kambin P, Casey K, O’Brien E, Zhou I: Transforaminal arthroscopic decompression of lateral recess stenosis. J Neurosurg 84: 462-467, 1996

9) Kambin P, Cohen LF, Brooks M, Schaffer JL: Development of degenerative spondylosis of the lumbar spine after partial discectomy. Comparison of laminotomy, discectomy, and posterolateral discectomy. Spine 20: 599-607, 1995

10) Kambin P, Gellman H: Percutaneous lateral discectomy of the lumbar spine: a preliminary report. Clin Orthop 174: 127-132, 1983

11) Kambin P, Savitz MH: Arthroscopic microdiscectomy: an alternative to open disc surgery. Mt Sinai J Med 67: 283-287, 2000

12) Kim WJ, Lim ST, Lee SH: Pyogenic psoas abscess and secondary spondylodiscitis as a rare complication of percutaneous endoscopic lumbar discectomy: a case report. Joint Diseases and Related Surgery 16: 163166, 2005

13) Knight MT, Ellison DR, Goswami A, Hillier VF: Review of safety in endoscopic laser foraminoplasty for the management of back pain. J Clin Laser Med Surg 19: 147-157, 2001

14) Knight MT, Goswami A, Patko JT, Buxton N: Endoscopic foraminoplasty: a prospective study on 250 consecutive patients with independent evaluation. $J$ Clin Laser Med Surg 19: 73-81, 2001

15) Lee DY, Ahn Y, Lee SH: Percutaneous endoscopic lumbar discectomy for adolescent lumbar disc herniation: surgical outcomes in 46 consecutive patients. Mt Sinai J Med 73: 864-870, 2006

16) Lee S, Kim SK, Lee SH, Kim WJ, Choi WC, Choi G, Shin SW: Percutaneous endoscopic lumbar discectomy for migrated disc herniation: classification of disc migration and surgical approaches. Eur Spine J 16: 431-437, 2007

17) Lee SH, Kang BU, Ahn Y, Choi G, Choi YG, Ahn KU, Shin SW, Kang HY: Operative failure of percutaneous endoscopic lumbar discectomy: a radiological analysis of 55 cases. Spine 31: E285-E290, 2006

18) Lew SM, Mehalic TF, Fagone KL: Transforaminal percutaneous endoscopic discectomy in the treatment of far-lateral and foraminal lumbar disc herniations. J Neurosurg 94: 216-220, 2001

19) Lonner BS, Scharf C, Antonacci D, Goldstein Y, Panagopoulos G: The learning curve associated with thoracoscopic spine instrumentation. Spine 30: 2835-2840, 2005

20) Mayer HM, Brock M: Percutaneous endoscopic lumbar discectomy (PELD). Neurosurg Rev 16: 115-120, 1993

21) Newton PO, Wenger DR, Mubarak SJ, Meyer RS: Anterior release and fusion in pediatric spinal deformity. A comparison of early outcome and cost of thoracoscopic and open thoracotomy approaches. Spine 22: 1398-1406, 1997
22) Pietilä TA, Stendel R, Kombos T, Ramsbacher J, Schulte $\mathrm{T}$, Brock M: Lumbar disc herniation in patients up to 25 years of age. Neurol Med Chir (Tokyo) 41: 340-344, 2001

23) Rantanen J, Hurme M, Falck B, Alaranta H, Nykvist F, Lehto M, Einola S, Kalimo H: The lumbar multifidus muscles five years after surgery for a lumbar intervertebral disc herniation. Spine 18: 568-574, 1993

24) Shim CS, Lee SH, Shin HD, Kang HS, Choi WC, Jung B, Choi G, Ahn Y, Lee S, Lee HY: CHARITE versus ProDisc: a comparative study of a minimum 3-year follow-up. Spine 32: 1012-1018, 2007

25) Yeung AT: The evolution of percutaneous spinal endoscopy and discectomy: state of the art. Mt Sinai J Med 67: 327-332, 2000

26) Yeung AT, Tsou PM: Posterolateral endoscopic excision for lumbar disc herniation: Surgical technique, outcome, and complications in 307 consecutive cases. Spine 27: 722-731, 2002

Address reprint requests to: Sang-Ho Lee, M.D., Department of Neurosurgery, Wooridul Spine Hospital, 47-4 Chungdam-dong Gangnam-gu, Seoul 135-100, R.O.K.

e-mail: shlee@wooridul.co.kr

\section{Commentary}

The authors present their experience with percutaneous lumbar endoscopic discectomy (PLED) for treatment of soft low lumbar disc herniation. The technique is well described and the results honestly reported. In my opinion, I do not see any significant advantages of PELD over open microdiscectomy. In recent years, anesthesiological and microsurgical procedures have been improved, postoperative pain is negligible, normal structures are preserved (just temporarily shifted), scar is minimal due to accurate hemostasis, and patients can be discharged the following day. Considering that the population has been carefully and correctly selected (and therefore these are the easiest cases) I find that also the operation time is comparable or longer than microdiscectomy, and that the complication rate and re-herniation are higher using PELD. I tried myself endoscopic discectomy 15 years ago, and I went back to microsurgery. Nevertheless, I find correct that always new attempts are made to improve the surgical technique, but I do not think that this will be the solution for the majority of patients with a lumbar herniated disc.

Alessandro Ducati, M.D. Ordinario di Neurochirurgia Universita' di Torino Torino, Italy 
Recently, endoscopically visualized procedures for lumbar disk herniation have been developed. These procedures were reportedly as effective as microdiscectomy, although there is no sufficient evidence based on randomized control study. These techniques are now highlighted because of the lower invasiveness for the normal anatomy of the dorsal musculature and ligaments, and will be widely accepted among neurosurgeons.

The authors presented surgical outcomes of percutaneous transforaminal endoscopic lumbar discectomy in a case series, and the steep learning curve for this procedure. Operative time was clearly shortened by experience. This technique appears to be safe and effective for selected single level intracanalicular soft disk herniation. Low complication rate and high success rate as well as the outstanding learning curve shown in this study will encourage neurosurgeons to introduce endoscopic lumbar disk surgery. Needless to say, sufficient experience in open surgery for lumbar disk is essential to understand the anatomical structures in a limited endoscopic view field. Indeed, the author experienced 200 cases of open microdiscectomy before starting endoscopic surgery. Furthermore, as mentioned by the authors, correct selection of patients is essential to obtain good surgical outcome. Cases with far migrated disk or with pathology at L5-S1 with high-positioned pelvic crest appear not to be good candidates.

Recurrence of herniation is one of the serious problems after surgery. The authors presented lower reherniation rates in Group II or III than in Group I, though no statistical difference was proven. This implies that this complication may be lessened by im- provement of surgical skill. I would like to know any tips to reduce recurrence of herniation, which were not mentioned in the paper. Further information in this regard will be helpful for the readers.

Toru HORIKOSHI, M.D. Department of Neurosurgery Interdisciplinary Graduate School of Medicine and Engineering University of Yamanashi Chuo, Yamanashi, Japan

The article of D. Y. Lee and S.-H. Lee describes a very important analysis of operated cases by percutaneous endoscopic lumbar discectomy (PELD). The method is firstly dependent on the indication for surgery in such cases. The inclusion criteria show an intracanalicular soft disk herniation in a single lumbar level L4-5 or L5-S1. The surgeon (D.Y.L.) has experience with open lumbar disk surgery of about 200 cases, which does not give the amount of experience that would be usual internationally. Of course the clearly defined study with the learning curve shows the advantage of using PELD. A mandatory part is to perform very exact radiographic and/or CT diagnostic. The paper does not describes exactly the endoscopic method used and the instruments. This would be important for the surgical view. Basically it is excellent that such a study has been made, showing the advantage of endoscopy use in this field.

Axel Perneczky, M.D.

The Neurosurgical Department of Medical School University of Mainz Mainz, Germany 